\title{
Fracture in Hierarchical Biomaterials: Human Hair
}

\author{
G. A. Camacho-Bragado*, G. Balooch**, C. Porter*, F. M. Dixon* and H. Bryant* \\ * L Oréal Recherche, Institute for Ethnic Hair and Skin Research, Chicago, IL \\ ** L'Oréal Recherche, Clark, NJ
}

Composites are found in a number of load bearing elements used in different engineering applications including car body parts, offshore drills, space structures, etc [1]. In an attempt to produce high-performance materials with multiple-functionality, inspiration has been drawn from complex composites found in nature including mineralized materials like bone and nacre as well as keratinized materials such as horns, nails and hair [2, 3]. Complex biological materials exhibit high toughness and tolerance to flaws while presenting a low density. These properties are achieved in nature by intercalating soft and hard materials into complex-hierarchical arrays [4 and refs within]. Consequently, failure in biological and bio-inspired materials is not always easily understood and therefore their mechanical behavior and long-term reliability cannot be accurately predicted, which limits the uses of engineered bio-materials.

A model biological material with a complex architecture is human hair. In a simplified way, hair can be regarded as a hierarchical material with a composite core (cortex) bordered by an armor-like shell (cuticle). Tightly packed keratinized cells composed by organized microfibrils, which are embedded in a protein matrix (keratin associated protein matrix), form the cortex. These same elements, cuticle and cortex, microfibrils and matrix, can lead to different degrees of curl. Even though straight and curly hair have both the same building blocks they differ in geometrical parameters and mechanical behavior.

Straight hair, in general, presents nearly circular cross sections while curly hair has elliptical to beanshape cross sections. Studies of the tensile behavior of straight vs curly hair [5] indicate a high incidence of premature failure for curly hair that is absent in straight hair. Post-mortem analysis of the fractured specimens showed a step fracture in straight hair vs a slanted step fracture in curly hair (Fig. 1). The stress-strain curves indicated similar break stress and strain values for both specimens (Fig 2.), thus the analysis of post-mortem sections alone does not provide enough information to explain the mechanisms of fracture in hair with different curl pattern.

In this study, we used two high spatial resolution techniques, SEM and X-ray tomography (XTM) to observe changes in the interior and the surface of hair during a step-wise tensile test. A uniaxial tensile force was applied to individual hair fibers in-situ in the SEM chamber. Tomograms were captured after each load application. It was observed that in the absence of cuticular or surface damage, the location of the final fracture could not be inferred; similar cuticle splaying was observed along the entire fiber once the post-yield point had been surpassed. Post-mortem images of the specimens analyzed by SEM + XTM and tested in a step-wise fashion were no different than those of the specimens that underwent continuous test indicating that the testing procedure does not modify the fracture mechanism of hair.

The combination of XTM and SEM imaging allowed us to follow changes in the material leading to elucidation of the crack propagation paths in hair and an understanding of the influence of the mesoscopic architecture in the overall mechanical behavior of this complex biological material. 


\section{References}

[1] A. G. Mamalis et al., Compos. Struct., 30 (1995) 131

[2] A. Carpinteri and N. M Pugno, Int J Fract., 150 (2008) 221; S. Mitragotri and J Lahann, Nat Matls., 8 (2009) 15

[3] L. Tombolato et al., Acta Biomat., 6 (2010) 319

[4] M. A Meyers et al., Prog Mat. Sci., 53 (2008) 1

[5] A. McMichael, J. Inv. Dermat., 12 (2007)6
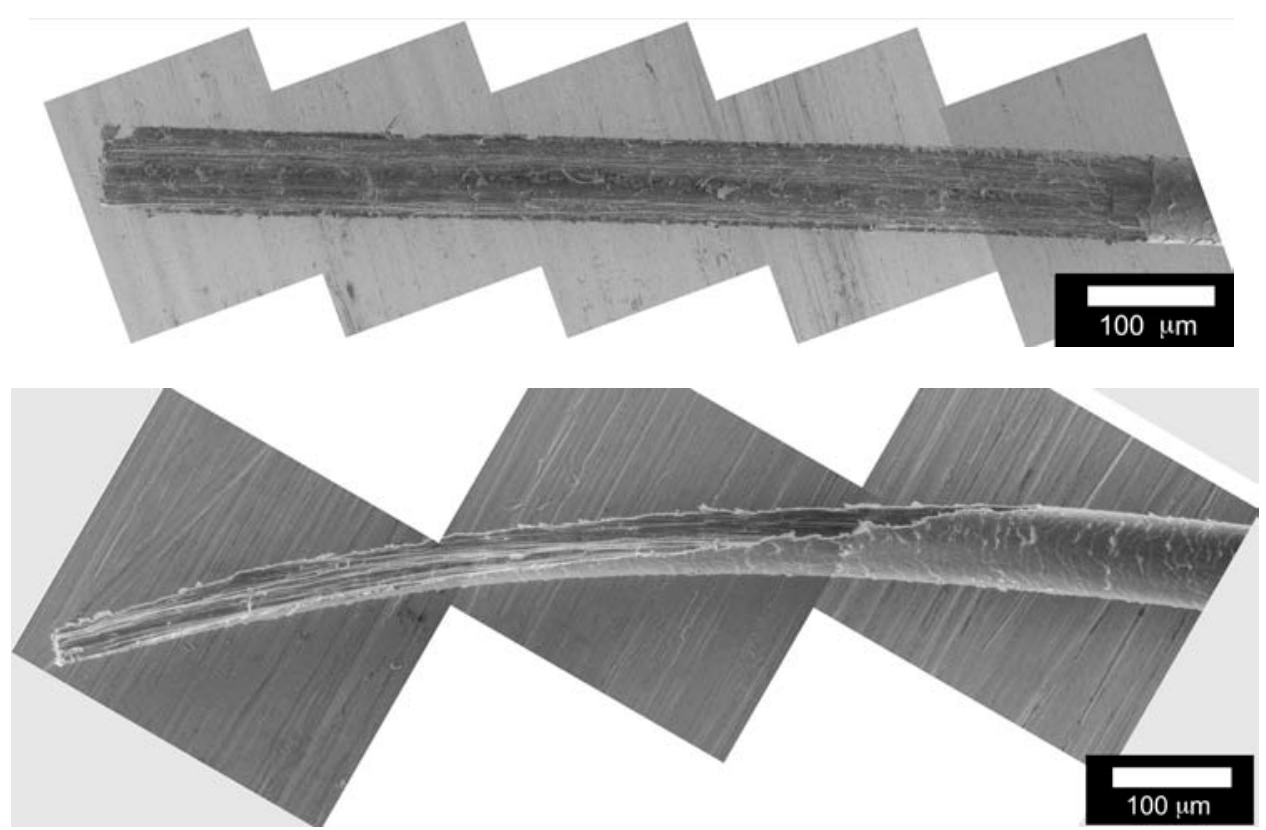

FIG. 1. Typical post-mortem images of straight (upper) and curly (lower) hair subjected to uniaxial tensile test.
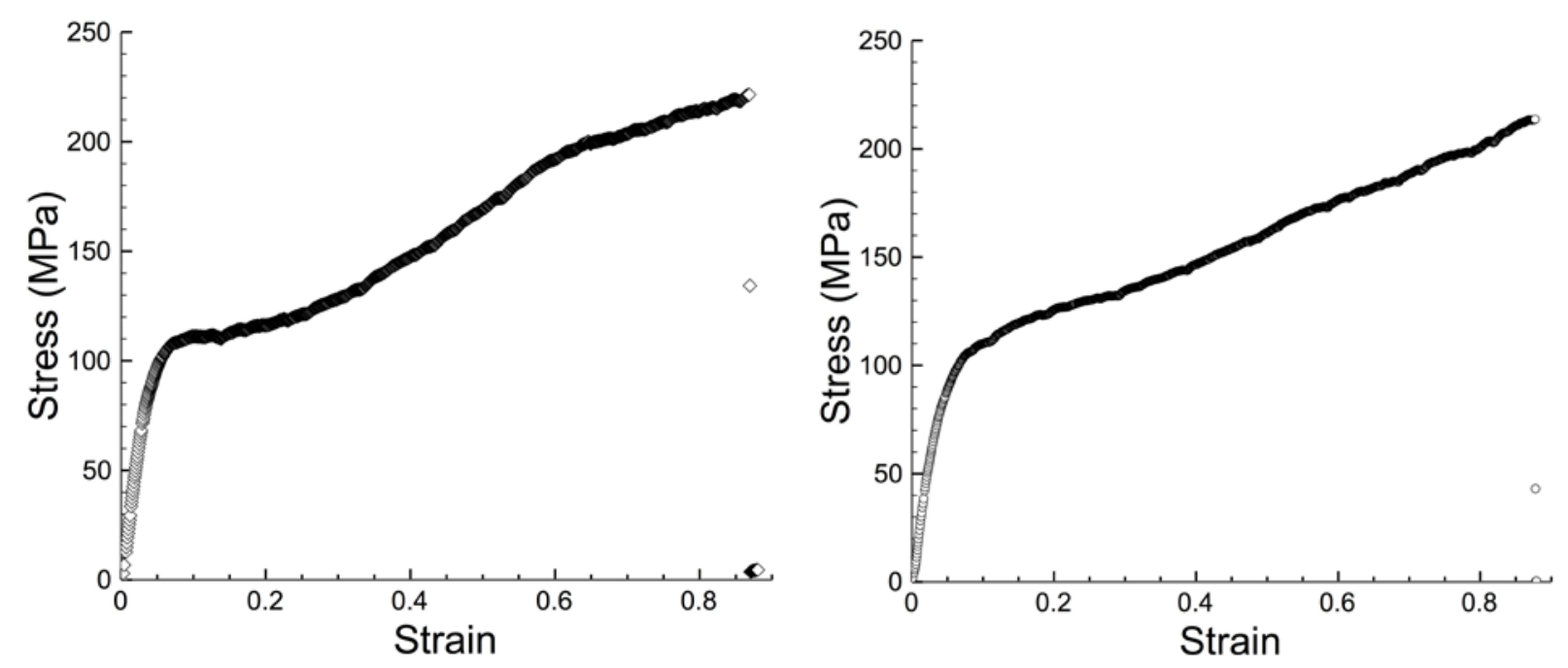

FIG. 2. Stress-strain curves for straight (right) and curly (left) hair taken at the same strain rates. 